\title{
Der männliche Familienlohn als Falle
}

\author{
ANNETTE DÜRING
}

\section{Löhne müssen zum Leben reichen}

Der Familienlohn, traditionell verstanden als Lohn eines männlichen Normalverdieners mit Vollarbeitszeit und beruflicher Qualifikation, ist längst nicht mehr die Norm - und war auch historisch nicht unbedingt die Norm. Gleichwohl gilt der Verdienst von Frauen - auch heute noch - oft als Zuverdienst zum männlichen Einkommen in der Familie.

Dieses Modell setzt für den Mann eine unbefristete langjährige Beschäftigung mit tarifvertraglich gesichertem Lohn voraus. Davon können wir allerdings nicht mehr ausgehen. Befristung, Leih arbeit, Werkverträge und Outsourcing mit verschlechterten Arbeitsbedingungen sind mittlerweile eher die Regel als die Ausnahme. Verlängerte Lebensarbeitszeiten verschärfen das Problem.

Andererseits arbeiten viele Frauen immer noch in typischen Frauenberufen im Erziehungs-, Pflege- und Dienstleistungsbereich mit Löhnen, die oft zum eigenständigen Leben kaum oder nicht ausreichen und zwangsläufig zu Altersarmut führen.

Zerbrechen Ehen oder Partnerschaften, verliert der Mann seinen Arbeitsplatz, ist er erwerbsunfähig oder prekär beschäftigt oder ist die Frau alleinerziehend, so werden Frauen freiwillig oder unfreiwillig zu Familienernährerinnen. Der typische Frauenlohn reicht dann zum Leben - erst recht mit Familie - nicht aus. Löhne müssen jedoch existenzsichernd sein, egal ob für Frauen oder Männer. Prekäre Beschäftigung gehört abgeschafft. Es darf nicht sein, dass Armutslöhne Lohnabhängige zu Hilfeempfängern im Sozialsystem machen und gleich zeitig die Profite von Unternehmen rasant ansteigen.

\section{Faire Bezahlung im Erziehungs-, Pflege- und Dienstleistungsbereich}

Frauen leisten wichtige Arbeit im vorschulischen Erziehungsbereich, in der Pflege älterer oder behinderter Menschen und im Dienstleistungsbereich. Gleichzeitig sind diese Arbeitsplätze schlecht bezahlt, werden oft nur in Teilzeit oder als Minijob angeboten und sind mit Schichtarbeit und ungüns- tigen Arbeitszeiten verbunden. Frauen stellen insgesamt zwei Drittel der prekär Beschäftigten. Wir setzen uns als Deutscher Gewerkschaftsbund (DGB) mit unseren Einzelgewerkschaften für faire, durch Tarifverträge abgesicherte Bezahlung dieser Arbeitsplätze ein. Bisher reichen die Tariflöhne im Erziehungs-, Pflege- und Dienstleistungsbereich allerdings nicht annähernd an die Löhne und Gehälter in der Metall- und Elektroindustrie oder im Banken- und Versicherungssektor heran. Frauenarbeit im Bereich der personenbezogenen Dienstleistungen wird - nach wie vor - deutlich schlechter bezahlt als insbesondere in technischen Bereichen. Hier liegen entscheidende gesellschafts- und tarifpolitische Aufgaben vor uns, zumal die Arbeitgeber im Erziehungs-, Pflege- und Dienstleistungsbereich in der Regel öffentliche Arbeitgeber sind.

\section{Entgeltlücke zwischen Frauen und Männern abschaffen - Arbeit und Einkommen gerecht verteilen}

Doch auch jenseits der frauentypischen Arbeitsbereiche verdienen Frauen immer noch ein Viertel weniger als Männer. Das gilt für viele Berufe und Branchen. Diese Tatsache ist ein Skandal. Die hohe Entgeltlücke (Gender Pay Gap) von Frauen in Deutschland zeichnet sich in Haushalten mit weiblichen Ernährerinnen besonders deutlich ab - und trifft damit nicht nur die Frauen, sondern auch deren Partner und Kinder. Die Bekämpfung der geschlechtsspezifischen Lohnlücke ist eine entscheidende gesellschaftspolitische Zielsetzung.

Es gibt ein weiteres Problem: Frauen verdienen in der Regel nicht nur weniger als Männer mit ihrer Erwerbsarbeit, sie leisten auch mehr unbezahl te Haus- und Fürsorgearbeit. Obwohl es heute mehr Familienernährerinnen gibt, werden sie in der Regel nicht oder nur wenig von ihren Partnern bei der Haus- und Fürsorgearbeit für Kinder und ältere Menschen entlastet. Diese Dominanz traditioneller Arbeitsteilung zwischen den Geschlechtern wird außerdem durch fehlende, unzureichende und teure Kinderbetreuungsangebote und unzureichende Ganztagsangebote im Schulbereich verstärkt. Arbeit und Einkommen müssen zwischen Männern und Frauen gerecht verteilt werden.

\section{Fehlanreize im Steuer- und Sozialrecht verändern}

Aktuell wird im Rahmen des Steuer- und Sozialrechts das Zuverdienst-Modell gefördert. Es sind zwar mehr Frauen erwerbstätig als vor zehn Jahren, allerdings oft in Minijobs, meist in Teilzeit und oft befristet. Die Befreiung von Steuern und Sozialabgaben im Minijob wird als passgenaues Angebot zum Wiedereinstieg für Familienfrauen ins Erwerbsleben angepriesen. Doch statt als Brücke in den sozialversicherungspflichtigen $\mathrm{Ar}$ beitsmarkt zu funktionieren, erweist sich der Minijob als Bumerang, der die Rückkehr in sozialversicherungspflichtige Beschäftigung verhindert. Gleichstellungspolitisch ist das MinijobModell eine Katastrophe. Daher müssen die Fehlanreize im Steuer- und Sozialrecht unbedingt verändert werden.

\section{Um,fair“teilen}

Gleichzeitig klafft gesamtgesellschaftlich die Schere zwischen Arm und Reich in Deutschland immer weiter auseinander. Allein die privaten Vermögen des reichsten Prozents der Deutschen sind höher als alle Schulden von Bund, Ländern und Kommunen zusammen. Das private Vermögen in Deutschland hat sich in den letzten zwanzig Jahren auf rund 10 Bill. $€$ mehr als verdoppelt. Mehr als die Hälfte dieses Vermögens gehört dabei nur $10 \%$ der Bevölkerung. Allein diese Zahlen zeigen: Die Zeit ist reif für echte Umverteilung! Eine Umverteilung zwischen Reich und Arm und zwischen Männern und Frauen.

\section{AUTORIN}

ANNETTE DÜRING, DGB Bremen-Elbe-Weser, Vorsitzende des Landesvorstandsbüros Bremen/Bremerhaven.

annette.duering@dgb.de 